\title{
Host cyclooxygenase-2 modulates carcinoma growth
}

\author{
Christopher S. Williams, ${ }^{1,2,3}$ Masahiko Tsujii, ${ }^{4}$ Jeff Reese, ${ }^{5}$ Sudhansu K. Dey, ${ }^{6}$ \\ and Raymond N. DuBois ${ }^{1,2,3,7}$ \\ ${ }^{1}$ Department of Medicine, \\ ${ }^{2}$ Department of Cell Biology, and \\ ${ }^{3}$ The Vanderbilt-Ingram Cancer Center, Vanderbilt University Medical Center, Nashville, Tennessee, USA \\ ${ }^{4}$ Department of Medicine, Osaka University School of Medicine, Osaka, Japan \\ ${ }^{5}$ Department of Pediatrics, and \\ ${ }^{6}$ Department of Molecular and Integrative Physiology, University of Kansas Medical Center, Kansas City, Kansas, USA \\ ${ }^{7}$ Veterans Affairs Medical Center, Nashville, Tennessee, USA
}

Address correspondence to: Raymond N. DuBois, Department of Medicine/Gastroenterology; Medical Center North C-2104, Vanderbilt University Medical Center, 1161 21st Avenue South, Nashville, Tennessee 37232-2279, USA.

Phone: (615) 343-4747; Fax: (615) 343-6229; E-mail: raymond.dubois@mcmail.vanderbilt.edu.

Received for publication February 15, 2000, and accepted in revised form April 12, 2000.

Cyclooxygenase-2 (COX-2; Ptgs2) acts as a tumor promoter in rodent models for colorectal cancer, but its precise role in carcinogenesis remains unclear. We evaluated the contribution of host-derived COX-1 and COX-2 in tumor growth using both genetic and pharmacological approaches. Lewis lung carcinoma (LLC) cells grow rapidly as solid tumors when implanted in C57BL/6 mice. We found that tumor growth was markedly attenuated in $\mathrm{COX}-2^{-/-}$, but not $\mathrm{COX}-1^{-/-}$or wild-type mice. Treatment of wild-type C57BL/ 6 mice bearing LLC tumors with a selective COX-2 inhibitor also reduced tumor growth. A decrease in vascular density was observed in tumors grown in $\mathrm{COX-2^{-/ }}$ mice when compared with those in wild-type mice. Because COX-2 is expressed in stromal fibroblasts of human and rodent colorectal carcinomas, we evaluated $\mathrm{COX}_{-2} 2^{-/-}$mouse fibroblasts and found a $94 \%$ reduction in their ability to produce the proangiogenic factor, VEGF. Additionally, treatment of wild-type mouse fibroblasts with a selective COX- 2 inhibitor reduced VEGF production by $92 \%$.

J. Clin. Invest. 105:1589-1594 (2000).

\section{Introduction}

Numerous studies of rodent cancer models and in humans show that nonsteroidal anti-inflammatory drugs (NSAIDs) have antineoplastic properties. One known effect of NSAIDs is their ability to inhibit the key enzymes required for prostaglandin production (prostaglandin endoperoxide synthase or cyclooxygenase). Two isoforms of cyclooxygenase have been characterized, COX-1 and COX-2. Most of the preclinical evidence for the antineoplastic effects of NSAIDs is based on studies using animals that have either a genetic or a chemically induced predisposition to develop colon cancer (1). These type of experimental approaches lack the power to determine whether the antineoplastic effects of NSAIDs are specifically due to inhibition of cyclooxygenase, the result of other effects, or both. Oshima et al. (2) evaluated $\mathrm{COX}-2^{-/-}$mice to determine whether COX-2 contributed to adenoma formation. They found that when mice with a genetic predisposition for polyp formation were mated with $\mathrm{COX}-2^{-1}$ mice, tumor burden in the offspring was significantly reduced when compared with that of control mice, strongly implicating a role for COX-2 in tumor promotion. Others have shown that treatment with COX-2 inhibitors leads to a marked reduction in the growth of a variety of neoplasms including colon (3), head and neck (4), skin (5), and bladder (6). Recent clinical studies have indicated that the presence of COX- 2 in human lung and colon cancers is associated with a negative prognosis $(7,8)$. Therefore, COX-2 may play a wider role in carcinogenesis than was originally thought.

The data regarding the sublocalization of COX- 2 in solid tumors are conflicting. COX-2 is expressed in some colon carcinoma cell lines (9-11), and its expression can be induced in cultured rat intestinal epithelial cells by treatment with mitogens and tumor promoters (12). Elevated COX-2 expression has been reported in the epithelial component of adenomas in the multiple intestinal neoplasia (Min) mouse (13), azoxymethane-treated rat cancers (14), replication error repair-positive human carcinomas (15), and sporadic human colorectal cancers $(16,17)$. We and others have recently found that COX-2 expression is also elevated in the subepithelial component in adenomas from the Min mouse $(18,19)$, in carcinogen-induced colon cancers in mice (18), and in colorectal carcinomas from IL-10 $10^{-/}$mice (20). Some of the conflicting data regarding localization of COX-2 may be due to nonspecific binding of the polyclonal antibodies that were used for COX-2 immunostaining or a change in COX-2 expression patterns as adenomas progress to carcinomas. Also, it seems clear from one recent report that COX-2 expression is found in both the epithelial and stromal components of sporadic human colorectal cancers (19). Oshima et al., localized COX-2 reporter expression in polyps from $\mathrm{APC}^{\Delta 716}$ mice by 

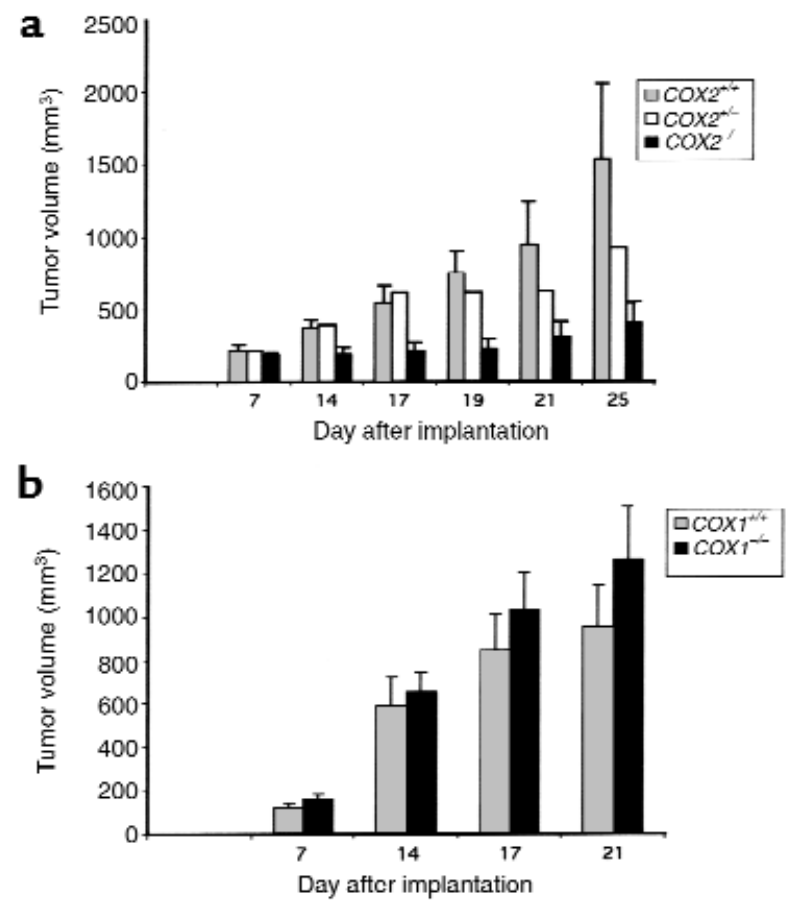

Figure 1

Host-derived COX-2 is important for LLC tumor growth. (a) A total of $2 \times 10^{6} \mathrm{LLC}$ cells were implanted into $\mathrm{COX}-2^{+/+}$(gray bars), COX$2^{+/-}$(white bars), or COX-2-/- (black bars) C57BL/ 6 mice; (b) a total of $2 \times 10^{6} \mathrm{LLC}$ cells were implanted into COX-1+/+ (gray bars) or COX$1^{-1-}$ (black bars) C57BL/6 mice. Tumor volumes were calculated from tumor measurements scored at the indicated day. Results are presented as the mean tumor volume \pm SEM.

crossing them with mice in which the LacZ gene was placed under the control of the COX-2 promoter (2). They found $L a c Z$ expression in areas surrounding the adenoma, but no significant expression in the epithelial component of the adenomas. We have recently carried out in situ hybridization experiments to localize COX-2 expression in sporadic colon cancers from 50 patients and in colon carcinomas from IL-10-/- mice. We found high levels of COX-2 mRNA expressed in stromal fibroblasts of both human and rodent colorectal cancers (20). It is clear from these results that COX-2 is expressed in both epithelial and tumor stromal cells. Therefore, it is possible that COX-2 in stromal fibroblasts could act to promote tumor growth by producing bioactive prostaglandins that have paracrine effects on nearby carcinoma cells. Recent reports have indicated that prostaglandins can regulate VEGF expression $(21,22)$ and that COX-2 inhibitors can directly affect angiogenesis (23). Others have shown that expression of VEGF by host fibroblasts plays an important role in angiogenesis (24). Therefore, COX-2 could be acting as a "landscaping tumor promoter" according to the model proposed by Kinzler and Vogelstein (25). This model supports the notion that COX-2 expression in the stromal component of a solid tumor could influence its growth or expression of proangiogenic factors. The mecha- nism(s) for inhibition of tumor formation and growth by NSAIDs may involve inhibition of COX-2 located in stromal tumor cells.

\section{Methods}

Reagents. Celecoxib (SC-58635) and SC-58125 were a kind gift from K. Siebert and P. Isakson of G.D. Searle and Co. (St. Louis, Missouri, USA).

Cell culture. Lewis lung carcinoma (LLC) cells were purchased from the American Type Tissue Collection (Manassas, Virginia, USA) and maintained according to standard cell culture techniques.

COX-1 and COX-2 knockout animals. The disruption of Ptgs2 (also referred to as COX-2) was performed by introducing a PGK-neo cassette in place of a $1.8-\mathrm{kb}$ EcoRV genomic fragment housing exon 1 and surrounding sequences (26). PCR of tail DNA and blood urea nitrogen (BUN) tests (26) determined the genotypes. The disruption of Ptgs 1 (also called COX-1) was performed by replacing $1 \mathrm{~kb}$ of intron 10 , together with the splice junction and first $44 \mathrm{bp}$ of exon 11, with the neomycin resistance gene (27). PCR analysis of tail DNA determined the genotypes.

Western blotting. Immunoblot analysis of cell protein lysates were performed as described previously (28). Briefly, tumor samples were lysed for 30 minutes in RIPA buffer $(1 \times$ PBS, $1 \%$ Nonidet P40, $0.5 \%$ sodium deoxycholate, $0.1 \%$ SDS). The clarified cell lysates (50 $\mu \mathrm{g})$ were denatured and fractionated by $10 \%$ SDSPAGE. The proteins were transferred to PVDF membranes after electrophoresis. The filters were blocked for 3 hours in BLOTTO $(0.15 \mathrm{M} \mathrm{NaCl}, 10 \mathrm{mM}$ Tris$\mathrm{HCl}$ [pH 7.4], 0.1\% Tween-20, and 5\% nonfat dry milk) and then incubated overnight with either COX-1 (1:500) or COX-2 (1:500) specific antibodies (COX-1 catalog no. 1753, COX-2 catalog no. 1746; Santa Cruz Biotechnology Inc., Santa Cruz, California, USA). The membranes were then washed in BLOTTO before a 1-

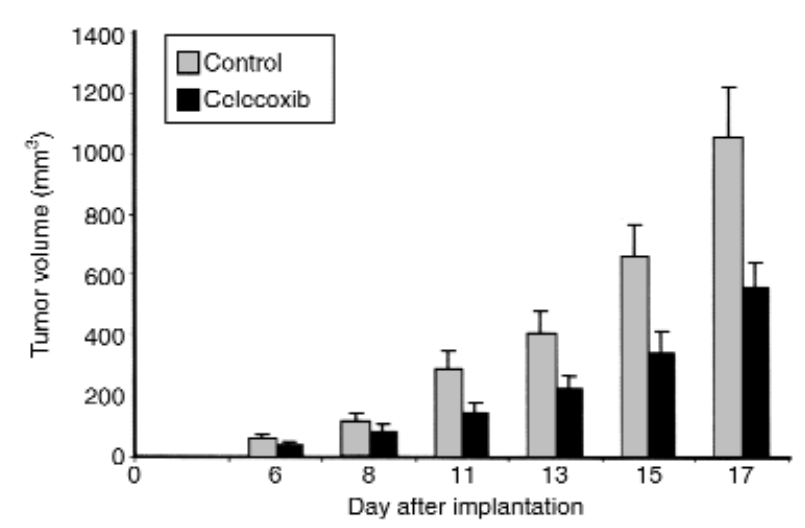

Figure 2

Celecoxib inhibits LLC tumor growth. A total of $2 \times 10^{6} \mathrm{LLC}$ cells were implanted into C57BL/ 6 mice. Mice were fed either control chow (gray bars) or $1,500 \mathrm{mg} / \mathrm{kg}$ celecoxib-containing chow (black bars). Tumor volumes were calculated from tumor measurements taken at the indicated day and are represented as the average of each group \pm SEM. 
a

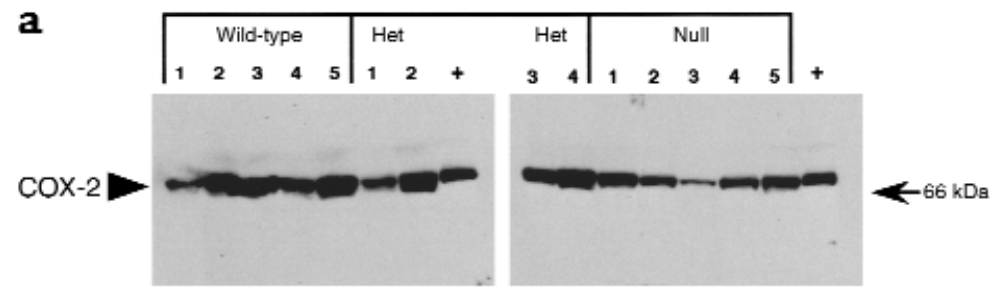

b
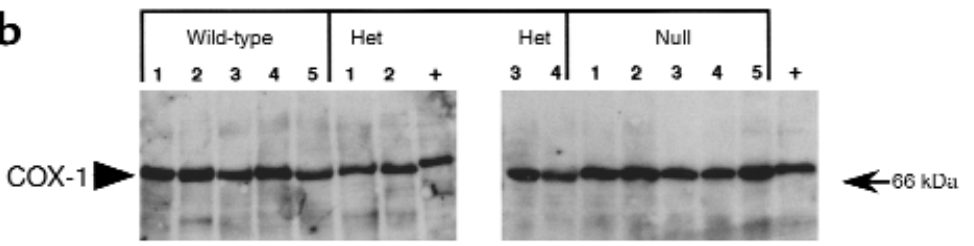

Figure 3

COX-1 and COX-2 are expressed within LLC tumors. Tumor lysates from LLC tumors grown in $\mathrm{COX}-2^{+/+}$ (wild-type), $\mathrm{COX}-2^{+/-}$(heterozygote), and $\mathrm{COX}-2^{-/-}$ (null) mice were produced and $50 \mu \mathrm{g}$ of lysate was fractionated on a $10 \%$ SDS-PAGE gel. (a) COX-2 and (b) COX-1 specific antisera were used to blot the membranes. Het, heterozygote. hour incubation with donkey anti-goat horseradish peroxidase-conjugated secondary antibody. The membranes were washed in TBST $(0.15 \mathrm{M} \mathrm{NaCl}, 10 \mathrm{mM}$ Tris- $\mathrm{HCl} \mathrm{pH} 7.4,0.1 \%$ Tween-20) and developed by the ECL plus chemiluminescence system (Amersham Life Sciences Inc., Arlington Heights, Illinois, USA) and exposed to Hyperfilm (Amersham Life Sciences).

Isograft model of tumor biology. LLC cells were grown on plastic culture dishes according to standard cell culture techniques (9), gently washed in sterile PBS, detached by reverse pipetting with PBS, and then pelleted by brief centrifugation at $300 \mathrm{~g}$. The supernatant was aspirated, and cells were resuspended in PBS and counted using a hemocytometer. A final concentration of $2 \times 10^{7}$ cells $/ \mathrm{mL}$ was made, and 100 $\mu \mathrm{L}$ of cell suspension was injected subcutaneously using a tuberculin syringe and a 27 -gauge needle. In celecoxib treatment experiments, mice were pretreated with celecoxib for 3 days before tumor implantation. Celecoxib was mixed in 4\% Harlan Teklad chow (Harlan Teklad, Madison, Wisconsin, USA) at $1,500 \mathrm{mg} / \mathrm{kg}$. The size of the tumor was determined by direct measurement of tumor dimensions. The volume was calculated according to the equation $\left(\mathrm{V}=\left[\mathrm{L} \times \mathrm{W}^{2}\right] \times 0.5\right)$, where $\mathrm{V}=$ volume, $\mathrm{L}=$ length, and $\mathrm{W}=$ width (29).

In situ bybridization. Sense or antisense [ $\left.{ }^{35} \mathrm{~S}\right]$-labeled cRNA probes were generated using appropriate polymerases from cDNAs to VEGF, COX-1, or COX-2 for in situ hybridization. The VEGF probe was derived from a VEGF cDNA fragment from nucleotides 270 to 849 of human VEGF. This fragment cross-hybridizes with murine VEGF (30). Mouse COX-1 and COX-2 probes were generated from murine cDNAs encompassing $\mathrm{Asn}_{82}-\mathrm{Gln}_{369}$ of COX-1 and $\mathrm{Met}_{1}-\mathrm{Gln}_{270}$ of COX-2 (31). The probes had specific activities of approximately $2 \times$ $10^{9}$ disintegrations per minute $/ \mu \mathrm{g}$. Sections hybridized with sense probes served as negative controls. After hybridization and washing, the slides were incubated with Rnase-A $(20 \mu \mathrm{g} / \mathrm{mL})$, and Rnase-A-resistant hybrids were detected by autoradiography using Kodak NTB-2 liquid emulsion (Eastman Kodak Co., Rochester, New York, USA).
Preparation of skin fibroblasts and VEGF determination. Skin tissue was chopped into small pieces and then placed into $60-\mathrm{mm}$ culture dishes and covered with DMEM $/ 20 \%$ FCS containing $0.01 \%$ collagenase. After 24 hours of culture, the tissue was washed and transferred into $10-\mathrm{cm}$ dishes containing DMEM/20\% FCS. After 10 days, spindle-shaped fibroblasts were collected and seeded at a 1:3 dilution. When these cells became $80 \%$ confluent, they were collected and counted, and 50,000 cells were seeded into $35-\mathrm{mm}$ culture dishes and incubated for 24 hours in DMEM/20\% FCS. The media were then changed, and serum-free DMEM was added to the cells and they were incubated for an additional 12 hours. The conditioned media were collected, and the

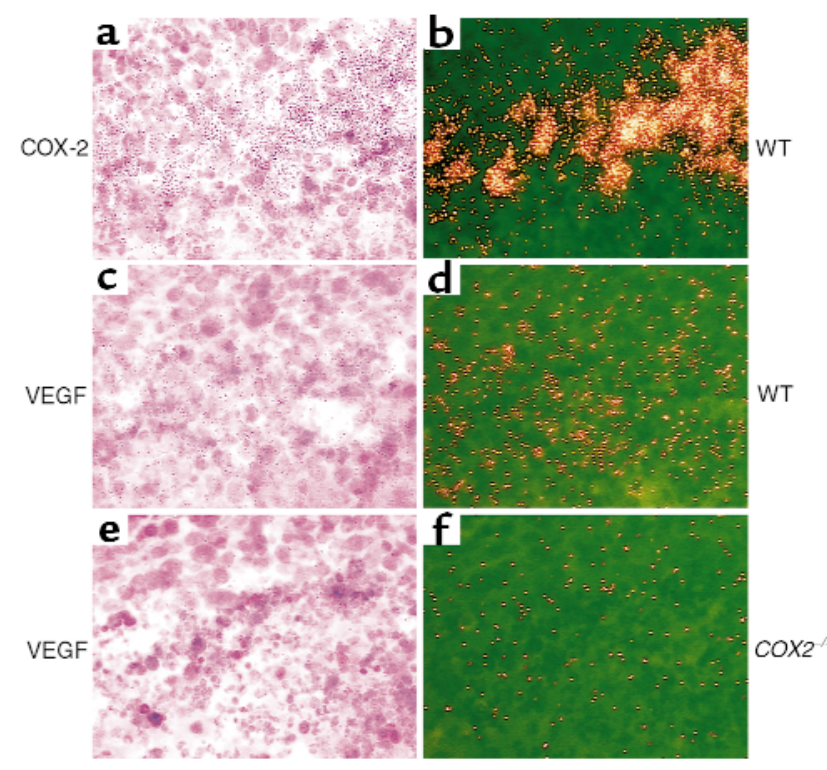

Figure 4

COX-2 and VEGF are expressed in LLC isografts. COX-2 (upper panels, $\mathbf{a}$ and $\mathbf{b}$, wild-type host), VEGF (middle panels, $\mathbf{c}$ and $\mathbf{d}$, wild-type host), and VEGF (lower panels, e and $\mathbf{f}, \mathrm{COX}-2^{-1-}$ host) riboprobes were hybridized to sections from tumors grown in C57BL/ 6 mice with the designated genotype $(\times 400)$. Control hybridizations with sense cRNA riboprobes were performed to validate the specificity of Rnase-A-resistant hybrids. WT, wild-type. 


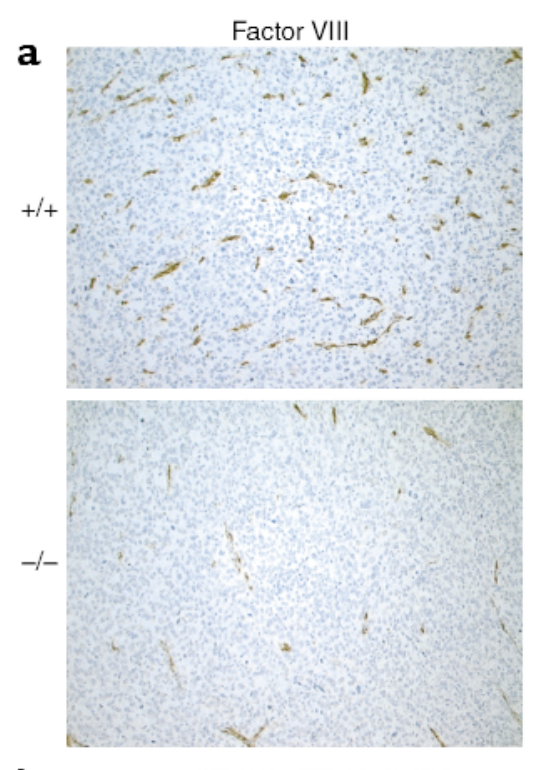

b

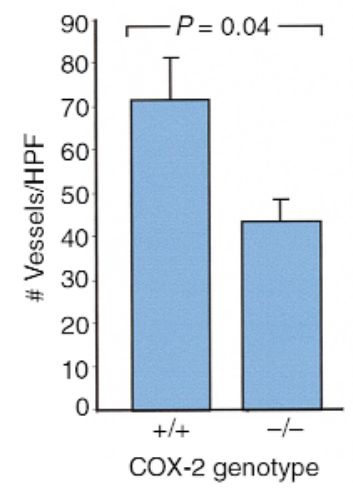

\section{Figure 5}

Decreased vascular density in LLC tumors when grown in COX-2-/mice. (a) Representative photomicrographs of factor VIII-stained tumor sections from LLC tumors grown in wild-type $(+/+)$ or Ptgs $2^{-1-}$ $(-/-)$ mice (×200). (b) Factor VIII-positive vessel counts obtained by morphometric analysis of LLC tumors. Values represent the average number of vessels at $\times 200 \pm$ the SD (Student's $t$ test; $P=0.04$ ). HPF, high power field.

concentration of VEGF was determined using a mouse VEGF immunoassay (catalog no. MMV00; R\&D Systems Inc., Minneapolis, Minnesota, USA).

Quantification of vascular density. The intratumoral blood vessels were quantitated using immunohistochemistry with a factor VIII rabbit anti-human polyclonal antibody (A0082; DAKO Corp., Carpinteria, California, USA). For each tumor, five random images were captured at $\times 200$. Only areas of viable tumor tissue were imaged; necrotic regions and overlying subdermal regions were excluded. Zeiss Image (Thornwood, New York, USA) morphometric analysis software was used to automatically count stained vessels, thus removing any possible observation bias. All tumors were imaged and analyzed using identical instrument settings over a 3hour period. Vessels that were visibly interconnected were scored only once. The final vascular density score for the tumor represents an average of all scored fields.

\section{Results and Discussion}

We were interested in carefully examining the role of host-derived COX-1 and COX-2 in tumor growth. We grafted LLC cells into either wild-type, COX-1-1-, or COX$2^{-1-}$ C57BL/6 mice. Using this model system, we directly tested the hypothesis that COX-1 or COX-2 produced by the host could affect tumor growth. We chose to study the LLC cells because they are capable of growing in the C57BL/6 mice without being rejected by the host, as they have a compatible genetic background; however, carcinoma cells derived from other species or mouse strains do not grow in these mice.

We found that the cyclooxygenase status of the host mouse does not influence tumor engraftment rates. All mice exhibited similar tumor growth rates during the first 7 days after implantation (Figure 1a). By day 15 , tumors grafted in the COX-2 null mice were significantly smaller than those engrafted in wild-type or $\mathrm{COX}-2^{+/-}$mice. In addition, tumors in $\mathrm{COX}-2^{-/-}$mice continued to grow more slowly than did control tumors for the remainder of the experiment. Interestingly, tumor growth in $\mathrm{COX}-2^{-/-}$mice increased slightly between days 20 and 25 , indicating that the absence of COX-2 in the host did not permanently halt carcinoma growth. No difference in the growth of LLC tumors in $\mathrm{COX}-1^{-/-}$mice was observed when compared with control mice (Figure 1b). This suggests that COX1 expression in the host is not essential for tumor growth under these conditions. Even though the lack of COX-1 is known to affect platelet function, there was no inhibition of tumor growth in the $\mathrm{COX}-1^{-/-}$ mice. Collectively, these data strongly implicate a role for host-derived COX-2 in promoting tumor growth in this model system.

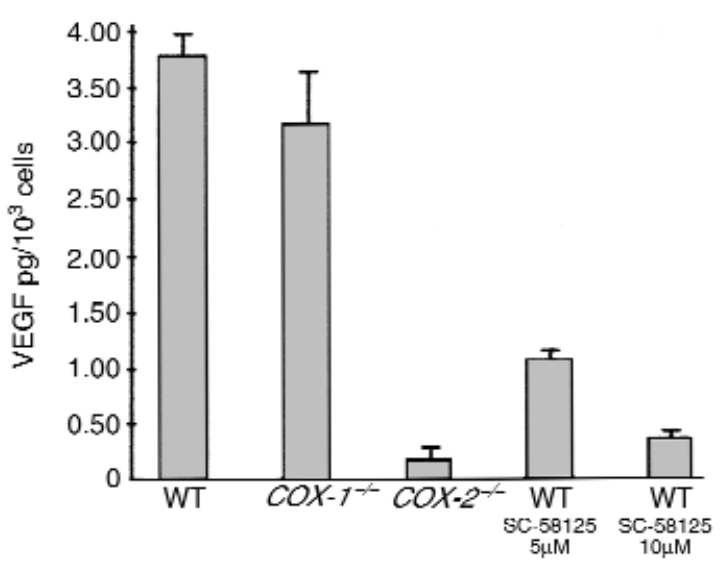

Figure 6

VEGF production in fibroblasts is regulated by COX-2. Production of VEGF by wild-type, $\mathrm{COX}-1^{-/-}$, and $\mathrm{COX}-2^{-/-}$fibroblasts was determined. Each treatment condition is listed below its respective bar graph. WT denotes wild-type mouse fibroblasts. We observed a $93 \%$ reduction in VEGF production in COX-2-/- fibroblasts when compared with wild-type fibroblasts. Additionally, treatment of wild-type fibroblasts with a selective COX-2 inhibitor (10 $\mu \mathrm{M}$ SC-58125) led to a $\sim 90 \%$ reduction in VEGF levels. 
We next evaluated whether treatment with celecoxib, a selective COX-2 inhibitor, could inhibit tumor growth. Celecoxib treatment was initiated 3 days before tumor implantation to mimic more closely the experiments conducted with the $\mathrm{COX}-2^{-/-}$animals. This treatment regimen would inhibit COX-2 activity in both the host and tumor tissues. Peak serum levels of celecoxib in treated mice were $889 \pm 272 \mathrm{ng} / \mathrm{mL}(2.3$ $\mu \mathrm{M})$. Celecoxib treatment inhibited tumor growth; however, the results were not as striking as those obtained in $\mathrm{COX}-2^{-/-}$mice (Figure 2 ). This variation may be due to differences between total lack of COX2 versus intermittently inhibiting COX-2 activity by treatment with dietary celecoxib.

To evaluate further LLC isografts, we examined intratumoral gene expression patterns. Cultured LLC cells express both COX-1 and COX-2. Immunoblotting of tumor lysates obtained from $\mathrm{COX}-2^{+/+}, \mathrm{COX}^{-2+/}$, or $\mathrm{COX}-2^{-/-}$mice did not reveal any differences in expression of either COX-1 or COX-2 (Figure 3). To determine whether differential localization of COX-1 or COX-2 expression occurs within the tumors, in situ hybridization for COX-1 and COX-2 was performed on tumor sections. The COX-1 hybridization pattern was diffuse, with uniform accumulation throughout the tumor (data not shown). COX-2 mRNA hybridization, in contrast, was focal with punctate accumulation in carcinoma and stromal cells that surrounded areas of necrotic tissue (Figure 4). Immunolocalization of COX-2 protein showed a similar expression pattern (data not shown). Interestingly, the expression pattern for $C O X-2$ and $V E G F$ was similar, with high levels of expression in the stromal compartment, and a significant reduction of $V E G F$ expression in tumors grown in the COX-2 $2^{-/-}$mice (Figure 4). We also examined the expression pattern of $C O X-2$ in human colorectal adenocarcinomas from 50 patients, using in situ hybridization, and found high levels of $C O X-2$ expressed in stromal fibroblasts of these tumors (data not shown).

Tumor growth requires the maintenance and expansion of a vascular network (32). It has been demonstrated using in vitro assays that COX-2 can influence angiogenesis $(23,33,34)$ and that treatment with selective COX-2 inhibitors blocks angiogenesis $(23,34,35)$. Therefore, we questioned whether vascularization of tumors grown in $\mathrm{COX}-2^{-/}$mice was affected by the lack of COX-2 expression in tumor host cells. We evaluated vascular density by selectively staining endothelial cells, as this is a measure of tumor-associated angiogenesis. We found that vascular density was 30\% lower in tumors grafted in $\mathrm{COX}-2^{-/-}$mice when compared with tumors from wild-type mice (Figure 5). From these results we conclude that COX-2 contributes to tumor vascularization. To explore the mechanism underlying this effect, we isolated fibroblasts from $\mathrm{COX}-1^{-/-}, \mathrm{COX}-2^{-/-}$, and wild-type mice and evaluated their ability to produce proangiogenic factors. We found that $\mathrm{COX}-2^{-/-}$fibroblasts have a $94 \%$ reduction in production of VEGF when compared with wild-type and $\mathrm{COX}-1^{-/-}$fibroblasts (Figure 6). Additionally, when wild-type fibroblasts were treated with a selective COX-2 inhibitor, we observed a $92 \%$ reduction in VEGF production (Figure 6). Both of these results demonstrate a link between COX-2 and regulation of VEGF expression. This is not so surprising in light of recent data indicating that the products of the COX-2 pathway (prostaglandins) can stimulate VEGF production $(21,22)$. Of interest, we found no difference in the levels of IGF in the fibroblasts isolated from COX$1^{-/-}, \mathrm{COX}-2^{-/-}$, or wild-type mice (data not shown). However, some groups have questioned the role of VEGF in maintaining the malignant phenotype of melanoma cells (36).

In summary, we have demonstrated that efficient tumor growth requires the presence of COX-2 in the tumor host. Our data also suggest that host-derived COX-2 may regulate intratumoral vascular density, which, in turn, may modulate tumor growth. We speculate that one mechanism for the antineoplastic effects of NSAIDs is via their inhibition of COX-2 located in the stromal compartment of the tumor, which leads to inhibition of the production of proangiogenic factors by tumor and stromal cells.

\section{Acknowledgments}

This work was supported in part by United States Public Health Services grants (DK 47297, P01CA77839, and P30 CA68485, to R.N. DuBois). R.N. DuBois is a recipient of a Veterans Affairs Research Merit Grant, and S.K. Dey is a recipient of an NICHD MERIT Award (HD 12304). We thank the T.J. Martell Foundation for their generous support. Thanks are due to Y. Zhu and N. Brown for genotyping of mice, and X. Zhao for in situ hybridization. We also thank Kay Washington for examining the histology of the tumors and Wade Krause for assistance with in vivo studies.

1. Williams, C.S., Mann, M., and DuBois, R.N. 1999. The role of cyclooxygenases in inflammation, cancer and development. Oncogene. 18:7908-7916.

2. Oshima, M., et al. 1996. Suppression of intestinal polyposis in Apc delta716 knockout mice by inhibition of cyclooxygenase 2 (COX-2). Cell. 87:803-809.

3. Kawamori, T., Rao, C.V., Seibert, K., and Reddy, B.S. 1998. Chemopreventive activity of celecoxib, a specific cyclooxygenase- 2 inhibitor, against colon carcinogenesis. Cancer Res. 58:409-412.

4. Nishimura, G., Yanoma, S., Mizuno, H., Kawakami, K., and Tsukuda, M. 1999. A selective cyclooxygenase-2 inhibitor suppresses tumor growth in nude mouse xenografted with human head and neck squamous carcinoma cells. Jpn. J. Cancer Res. 90:1152-1162.

5. Pentland, A.P., Schoggins, J.W., Scott, G.A., Khan, K.N., and Han, R. 1999. Reduction of UV-induced skin tumors in hairless mice by selective COX-2 inhibition. Carcinogenesis. 20:1939-1944.

6. Okajima, E., et al. 1998. Chemopreventive effects of nimesulide, a selective cyclooxygenase-2 inhibitor, on the development of rat urinary bladder carcinomas initiated by $N$-butyl- $N$-(4-hydroxybutyl) nitrosamine. Cancer Res. 58:3028-3031.

7. Achiwa, H., et al. 1999. Prognostic significance of elevated cyclooxygenase 2 expression in primary, resected lung adenocarcinomas. Clin. Cancer Res. 5:1001-1005.

8. Sheehan, K.M., et al. 1999. The relationship between cyclooxygenase-2 expression and colorectal cancer. JAMA. 282:1254-1257.

9. Sheng, H., et al. 1997. Inhibition of human colon cancer cell growth by selective inhibition of cyclooxygenase-2. J. Clin. Invest. 99:2254-2259. 
10. Hanif, R., et al. 1996. Effects of nonsteroidal anti-inflammatory drugs on proliferation and on induction of apoptosis in colon cancer cells by a prostaglandin-independent pathway. Biochem. Pharmacol. 52:237-245.

11. Coffey, R.J., et al. 1997. EGF receptor activation induces nuclear targeting of COX-2, basolateral release of prostaglandins and mitogenesis in polarizing colon cancer cells. Proc. Natl. Acad. Sci. USA. 94:657-662.

12. DuBois, R.N., Awad, J., Morrow, J., Roberts, L.J., and Bishop, P.R. 1994. Regulation of eicosanoid production and mitogenesis in rat intestinal epithelial cells by transforming growth factor- $\alpha$ and phorbol ester. J. Clin. Invest. 93:493-498.

13. Williams, C.S., et al. 1996. Elevated cyclooxygenase-2 levels in Min mouse adenomas. Gastroenterology. 111:1134-1140.

14. Shao, J., et al. 1999. Coordinate regulation of cyclooxygenase-2 and TGF$\beta 1$ in hereditary nonpolyposis colorectal cancer and azoxymethaneinduced rat colonic tumors. Carcinogenesis. 20:185-191.

15. Karnes, W.E., et al. 1998. Reduced COX-2 protein in colorectal cancer with defective mismatch repair. Cancer Res. 58:5473-5477.

16. Kutchera, W., et al. 1996. Prostaglandin H synthase-2 is expressed abnormally in human colon cancer: evidence for a transcriptional effect. Proc. Natl. Acad. Sci. USA. 93:4816-4820.

17. Sano, H., et al. 1995. Expression of cyclooxygenase-1 and -2 in human colorectal cancer. Cancer Res. 55:3785-3789.

18. Shattuck-Brandt, R.L., Lamps, L.W., Heppner-Goss, K.J., DuBois, R.N., and Matrisian, L.M. 1999. Differential expression of matrilysin and cyclooxygenase- 2 in intestinal and colorectal neoplasms. Mol. Carcinog. 24:177-187.

19. Chapple, K.S., et al. 2000. Localization of cyclooxygenase- 2 in human sporadic colorectal adenoma. Am. J. Pathol. 156:545-553.

20. Shattuck-Brandt, R.L., et al. 2000. Cyclooxygenase-2 expression is increased in the subepithelial myofibroblasts of colon and cecal carcinomas from IL-10 (-/-) mice. Gastroenterology. 118:337-345.

21. Cheng, T., Cao, W., Wen, R., Steinberg, R.H., and LaVail, M.M. 1998. Prostaglandin $\mathrm{E}_{2}$ induces vascular endothelial growth factor and basic fibroblast growth factor mRNA expression in cultured rat Muller cells. Invest. Ophthalmol. Vis. Sci. 39:581-591.

22. Hoper, M.M., et al. 1997. Prostaglandins induce vascular endothelial growth factor in a human monocytic cell line and rat lungs via cAMP. Am. J. Respir. Cell Mol. Biol. 17:748-756.
23. Jones, M.K., et al. 1999. Inhibition of angiogenesis by nonsteroidal antiinflammatory drugs: insight into mechanisms and implications for cancer growth and ulcer healing. Nat. Med. 5:1418-1423.

24. Fukumura, D., et al. 1998. Tumor induction of VEGF promoter activity in stromal cells. Cell. 94:715-725.

25. Kinzler, K.W., and Vogelstein, B. 1998. Landscaping the cancer terrain. Science. 280:1036-1037.

26. Dinchuk, J.E., et al. 1995. Renal abnormalities and an altered inflammatory response in mice lacking cyclooxygenase II. Nature. 378:406-409.

27. Langenbach, R., et al. 1995. Prostaglandin synthase-1 gene disruption in mice reduces arachidonic acid induced inflammation and indomethacin induced gastric ulceration. Cell. 83:483-492.

28. DuBois, R.N., Shao, J., Sheng, H., Tsujii, M., and Beauchamp, R.D. 1996. G1 delay in intestinal epithelial cells overexpressing prostaglandin endoperoxide synthase-2. Cancer Res. 56:733-737.

29. Wang, J., et al. 1995. Demonstration that mutation of the type II transforming growth factor beta receptor inactivates its tumor suppressor activity in replication error-positive colon carcinoma cells. J. Biol. Chem. 270:22044-22049.

30. Chakraborty, I., Das, S.K., and Dey, S.K. 1995. Differential expression of vascular endothelial growth factor and its receptor mRNAs in the mouse uterus around the time of implantation. J. Endocrinol. 147:339-352.

31. Chakraborty, I., Das, S.K., Wang, J., and Dey, S.K. 1996. Developmental expression of the cyclo-oxygenase- 1 and cyclo-oxygenase-2 genes in the peri-implantation mouse uterus and their differential regulation by the blastocyst and ovarian steroids. J. Mol. Endocrinol. 16:107-122.

32. Holash, J., et al. 1999. Vessel cooption, regression, and growth in tumors mediated by angiopoietins and VEGF. Science. 284:1994-1998.

33. Tsujii, M., et al. 1998. Cyclooxygenase regulates angiogenesis induced by colon cancer cells. Cell. 93:705-716.

34. Daniel, T.O., Liu, H., Morrow, J.D., Crews, B.C., and Marnett, L.J. 1999. Thromboxane $\mathrm{A}_{2}$ is a mediator of cyclooxygenase-2-dependent endothelial migration and angiogenesis. Cancer Res. 59:4574-4577.

35. Yamada, M., Kawai, M., Kawai, Y., and Mashima, Y. 1999. The effect of selective cyclooxygenase- 2 inhibitor on corneal angiogenesis in the rat. Curr. Eye Res. 19:300-304.

36. Chin, L., et al. 1999. Essential role for oncogenic Ras in tumour maintenance. Nature. 400:468-472. 